\title{
Post-endoscopic retrograde cholangiopancreatography pneumomediastinum caused by probing a pseudopapilla: Case report of a rare complication treated conservatively
}

\author{
Justin Michael Cloutier, Donald Rudy Duerksen
}

\begin{abstract}
Introduction: Pneumomediastinum and subcutaneous emphysema have rarely been reported to occur as a result of duodenal perforation from Endoscopic retrograde cholangiopancreatography (ERCP) and sphincterotomy. Case Report: In this report, we describe a case of pneumomediastinum associated with ERCP. There was no evidence of duodenal or esophageal perforation on imaging and the patient was treated conservatively with full recovery. Conclusion: In the absence of frank perforation, pneumomediastinum and subcutaneous emphysema are rare complications of ERCP that can be managed conservatively with fasting, intravenous fluids and antibiotics.
\end{abstract}

Keywords: Endoscopic retrograde cholangiopancreatography (ERCP) complications, Esophageal perforation, Pneumomediastinum

Justin Michael Cloutier ${ }^{1}$, Donald Rudy Duerksen ${ }^{2}$

Affiliations: ${ }^{1} \mathrm{MD}$, B.Sc, Department of Internal Medicine, University of Manitoba, Winnipeg, MB; ${ }^{2} \mathrm{MD}$ FRCPC, Department of Internal Medicine, Section of Gastroenterology, University of Manitoba, Winnipeg, MB.

Corresponding Author: Dr. Donald R Duerksen, Department of Medicine, Division of Gastroenterology, C5120 409 Tache Ave, St. Boniface Hospital Winnipeg, Manitoba, Canada R2H 2A6; Ph: (204) 237-2796; Fax: (204) 233-7154; Email: duerksn@cc.umanitoba.ca

Received: 05 April 2015

Accepted: 03 July 2015

Published: 03 September 2015

\section{How to cite this article}

CloutierJM,DuerksenDR.Post-endoscopicretrograde cholangiopancreatography Pneumomediastinum Caused by Probing a pseudopapillary: Case report of a rare complication treated conservatively. Int $\mathrm{J}$ Hepatobiliary Pancreat Dis 2015;5:82-85.

Article ID: 100039IJHPDJC2015

$* * * * * * * * *$

doi:10.5348/ijhpd-2015-39-CR-14

\section{INTRODUCTION}

Pneumomediastinum is a rare complication of endoscopic retrograde cholangiopancreatography (ERCP) that may signify frank duodenal perforation. In this report, we describe a case of post-ERCP pneumomediastinum successfully managed conservatively with a brief period of fasting and intravenous fluid support.

\section{CASE REPORT}

An 87-year-old female with a longstanding history of abdominal pain underwent magnetic resonance cholangiopancreatography which demonstrated changes consistent with chronic pancreatitis, including a pancreatic duct stone in the main pancreatic duct. Her past medical history was significant for bladder surgery and a hernia repair. She was not on medications. There was no history of ethanol abuse. At a subsequent ERCP 
she had a 'pseudopapilla' (Figure 1) which was initially probed with a sphincterotome and guide-wire. However, it was not possible to advance the guide-wire more than 2-3 mm. It was then noted that her true papilla was hidden under a duodenal fold - this was probed with a cannula and the common bile duct was selectively cannulated with a guidewire. A cholangiogram was obtained and was normal. Before the cannula could be repositioned in an attempt to cannulate the pancreatic duct, it was noted that she had developed some neck swelling and probable subcutaneous emphysema. The procedure was terminated and an urgent computed tomography (CT) scan confirmed a pneumomediastinum with subcutaneous emphysema (Figures 2 and 3). A water soluble contrast study of the upper gastrointestinal tract did not demonstrate any evidence of extravasation of contrast. She complained of mild chest and neck discomfort as well as nausea. She had no difficulty breathing but noted that here voice had changed and had a higher pitch. She did not note any difficulty swallowing her own saliva. On physical examination she had evidence of neck crepitus. Her lungs were clear to auscultation and her heart sounds were normal. Her oxygen saturation on 4L was $100 \%$. She was admitted to hospital where she was treated conservatively with observation, nil per oral and intravenous antibiotics, (ceftriaxone and metronidazole). She was provided intravenous fluids (dextrose and half normal saline at a rate of $100 \mathrm{ml} / \mathrm{h}$ ) and was treated with intravenous ondansetron for nausea. Her nausea and chest and neck discomfort improved over the first three days of her admission. She did not develop any fever or pulmonary symptoms and her antibiotics were discontinued after four days. Her subcutaneous emphysema resolved. The patient was started on clear fluids on day-3 and discharged on a regular diet on day-5. At follow-up after two weeks, she remained asymptomatic. A subsequent MRI scan showed complete resolution of the pneumomediastinum.

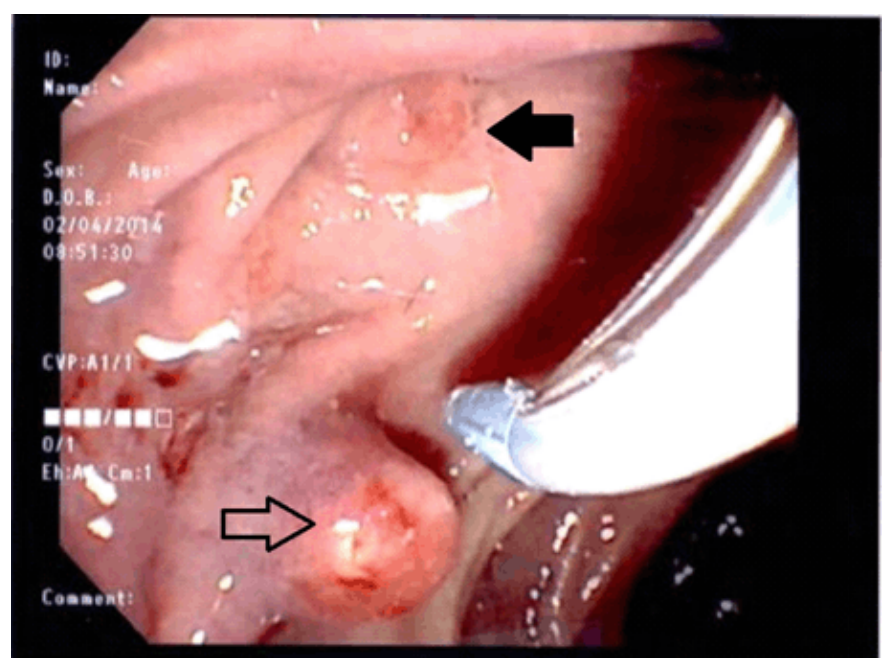

Figure 1: 'Pseudopapilla' (clear arrow), probed on endoscopy with major papilla (solid arrow), nearby. The major papilla was cannulated and the common bile duct was normal.

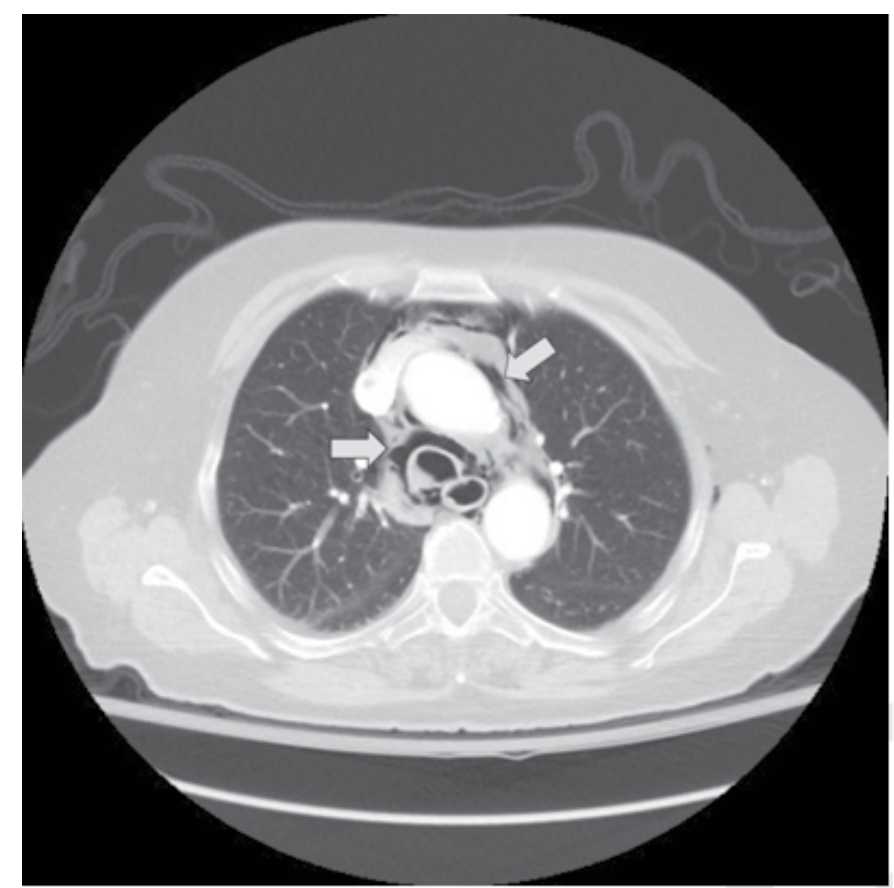

Figure 2: Computed tomography scan after Endoscopic retrograde cholangiopancreatography (ERCP) demonstrating pneumomediastinum (arrow).

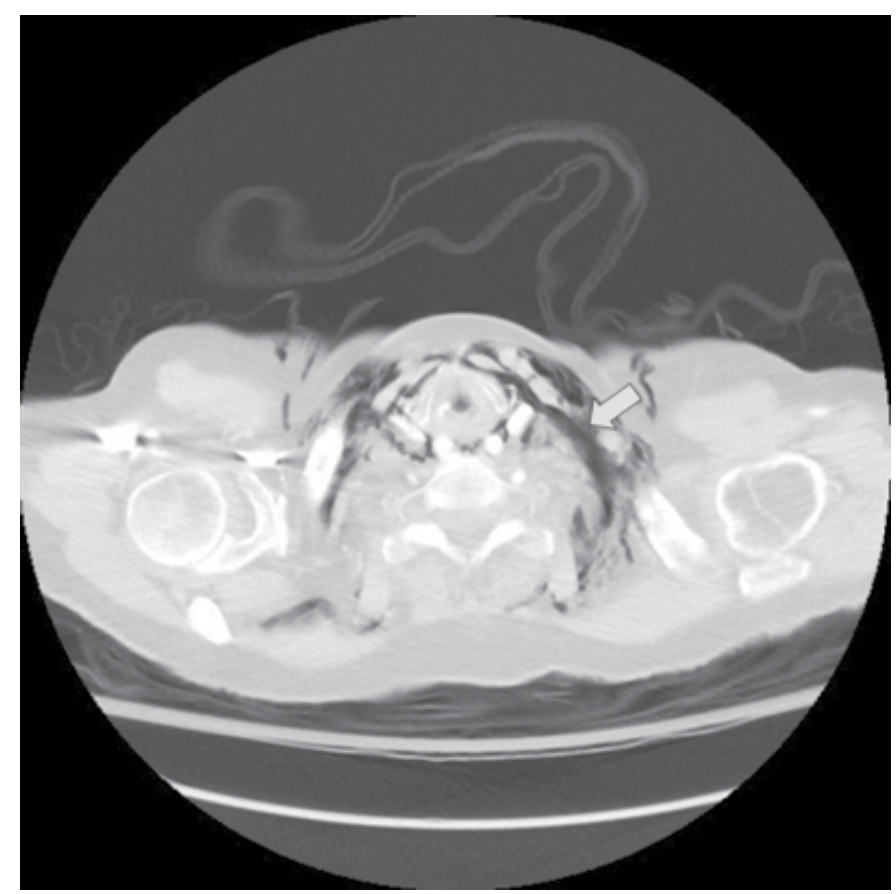

Figure 3: Computed tomography scan after Endoscopic retrograde cholangiopancreatography (ERCP) demonstrating subcutaneous emphysema (arrow).

\section{DISCUSSION}

The presence of retroperitoneal air after ERCP has been well described and in the absence of significant physical findings of free perforation, requires no specific intervention [1]. Pneumothorax, pneumomediastinum and subcutaneous emphysema have also been reported in association with ERCP and sphincterotomy [2]. The 
constellation of pneumomediastinum with subcutaneous emphysema without pneumothorax has been described in one other case report [3], and in that report the patient was also successfully managed with conservative care. In that reported case, the 83-year-old patient had a similar presentation of subcutaneous emphysema following an ERCP and sphincterotomy for acute cholangitis. She was otherwise stable and had no evidence of luminal perforation with gastrograffin imaging. Her subcutaneous emphysema resolved within four days, similar to the response in our case.

It is thought that this unique complication arises when sphincterotomy (in our case, probing the pseudopapilla) compromises the luminal integrity, and insufflation causes transudation of air across the mucosa to spaces such as the retroperitoneal space and the mediastinum. A continuum of fascial planes connects cervical soft tissues with the mediastinum, causing subcutaneous emphysema, and rapid transfer of air across these spaces [3].

Post-ERCP pneumomediastinum, pneumothorax, or pneumoperitoneum that is associated with free perforation often requires surgical repair [4]. In the absence of frank perforation (as in this case) patients may be managed conservatively with a period of fasting, intravenous fluids, antibiotics, and chest tube drainage, if required.

\section{CONCLUSION}

Post-endoscopic retrograde cholangiopancreatography (post-ERCP) pneumomediastinum may be caused by probing the duodenum with a sphincterotome. In the absence of frank perforation, this case report suggests that conservative management can result in complete resolution.

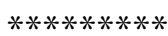

\section{Author Contributions}

Justin Michael Cloutier - Substantial contributions to conception and design, Acquisition of data, Analysis and interpretation of data, Drafting the article, Revising it critically for important intellectual content, Final approval of the version to be published

Donald Rudy Duerksen - Analysis and interpretation of data, Revising it critically for important intellectual content, Final approval of the version to be published

\section{Guarantor}

The corresponding author is the guarantor of submission.

\section{Copyright}

(C) 2015 Justin Michael Cloutier et al. This article is distributed under the terms of Creative Commons Attribution License which permits unrestricted use, distribution and reproduction in any medium provided the original author(s) and original publisher are properly credited. Please see the copyright policy on the journal website for more information.

\section{REFERENCES}

1. Genzlinger JL, McPhee MS, Fisher JK, Jacob KM, Helzberg JH. Significance of retroperitoneal air after endoscopic retrograde cholangiopancreatography with sphincterotomy. Am J Gastroenterol 1999 May;94(5):1267-70.

2. Schepers NJ, van Buuren HR. Pneumothorax following ERCP: report of four cases and review of the literature. Dig Dis Sci. 2012 Aug;57(8):1990-5.

3. Papamichail M, Nikolaidis N, Anastasiou E, Sidirokastritis G, Prigouris P. Massive Subcutaneus Emphysema following Endoscopic Retrograde Cholangiopancreatography with Sphincterotomy. Case Rep Gastroenterol 2010 Sep 20;4(3):399-403.

4. Miller R, Zbar A, Klein Y, et al. Perforations following endoscopic retrograde cholangiopancreatography: a single institution experience and surgical recommendations. Am J Surg 2013 Aug;206(2):1806.

\section{Conflict of Interest}

Authors declare no conflict of interest. 
Access full text article on other devices

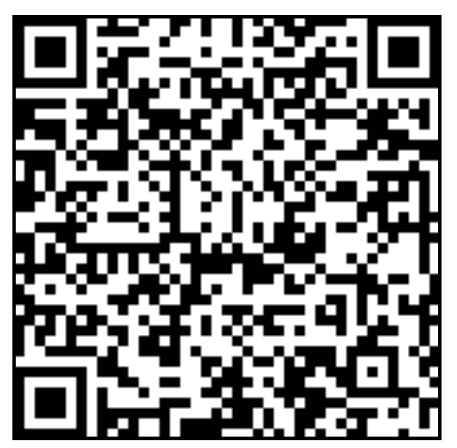

Access PDF of article on other devices

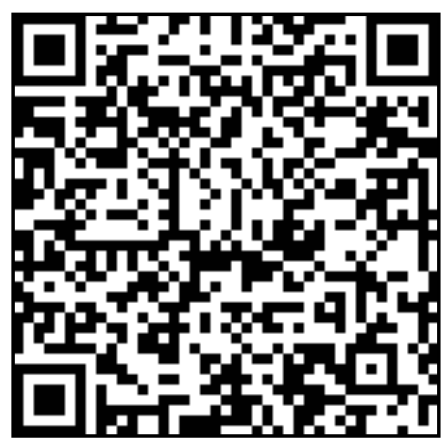

